\title{
Efficacy and safety of mini percutaneous nephrolithotomy in obese patients
}

\author{
Fatih Akbulut*, Onur Kucuktopcu, Emre Kandemir, Burak Ucpinar, Faruk Ozgor, Abdulmuttalip Simsek, \\ Burak Arslan, Akif Erbin, Fatih Yanaral, Murat Binbay and Gokhan Gurbuz
}

\begin{abstract}
Purpose: We aimed to evaluate the effect of obesity on surgical outcomes of mini percutaneous nephrolithotomy (Mini-PNL).

Methods: Hundred and eighty two Mini-PNL procedures were performed between May 2013 and January 2015 and their results were evaluated retrospectively. Patients were classified as non-obese (BMI, $\left.18.5-30 \mathrm{~kg} / \mathrm{m}^{2}\right)$ and obese $\left(\geq 30 \mathrm{~kg} / \mathrm{m}^{2}\right)$ groups. Obese and non-obese patients were compared according to pre-operative demographic values, intra-operative surgery techniques and post-operative results.

Results: BMI values of 133 patients were lower than $30 \mathrm{~kg} / \mathrm{m}^{2}$ while 49 patient's BMI values were higher than $30 \mathrm{~kg} /$ $\mathrm{m}^{2}$. There were no significant difference between operation time, fluoroscopy time, number of access and access sites when two groups were compared. No significant difference was found in total length of hospital stay, hemoglobin drop, and complication rates. Stone-free rates were $70.7 \%$ in the non-obese and $71.4 \%$ in the obese group ( $p=0.9$ ).

Conclusions: Mini-PNL procedure is a safe and effective treatment modality, which should be strongly considered for obese patients with appropriate sized stones.
\end{abstract}

Keywords: Mini percutaneous nephrolithotomy, Obesity, Surgical outcomes, Complications, Safety

\section{Background}

Obesity is a serious health problem that arises due to world's changing social and economic status and physical inactivity (Shikora 2005). Obese patients have increased risk of having diseases like; diabetes mellitus, hypertension, cardiovascular diseases, obstructive sleep apnea syndrome, and urinary stone disease (Taylor et al. 2005; Stamatelou et al. 2003; Curhan et al. 1998). Increased insulin resistance, hyperinsulinemia, hypocitraturia, hyperoxaluria and hypercalcemia can be listed as possible causes of increased incidence of stone disease among obese patients (Goldfarb 2003; Yang and Bellman 2004; Curhan 2007). Thus, treatment of stone disease in obese patients may be more important health issue in the near future.

\footnotetext{
*Correspondence: drfakbulut@hotmail.com Department of Urology, Haseki Training and Research Hospital, Istanbul, Turkey
}

Shock wave lithotripsy (SWL) treatment in obese patients appears to be less effective due to increased skin-to-stone distance and difficulties in performing the procedure (Thomas and Cass 1993; Al-Hayek et al. 2013). Performing flexible URS (F-URS) in obese patients with high stone burden can result in increased amount of residual stone fragments (Fabrizio et al. 1998). Residual stone fragments may be an obstacle to optimal treatment of obese patients who have increased risk of having stone disease.

Percutaneous nephrolithotomy is a commonly performed procedure in obese patients, even though it has certain problems like; bleeding, pain, and difficulties in positioning of the patient. Although several studies indicate that PNL is a reasonable option in obese patients (Faerber and Goh 1997; Carson et al. 1988; El-Assmy et al. 2007; Koo et al. 2004), there are also studies, which show increased risk of bleeding, technical difficulties in patient positioning, and difficulties in surgery techniques (Bagrodia et al. 2008; Desai et al. 2004; Sergeyev et al. 2007; Olbert et al. 2007). 
Bleeding which requires transfusion due to conventional PNL procedure is one of the serious problems that urologists encounter. Transfusion rates can be as high as $21 \%$ in morbid obese patients (Pearle et al. 1998). According to one study, tract dilatation technique and diameter of the tract are listed as factors that affect the risk of bleeding (Kukreja et al. 2004). After the invention of mini-PNL, the size of working sheaths decreased. This process resulted in decreased hemorrhage-related complications and hospitalization times with similar stone-free rates. Due to these reasons; mini-PNL procedure, which is performed through a narrower and shorter tract, is preferred in appropriate cases (Helal et al. 1997). Today, with the development of different size instruments there is a confusion of terminology used to describe the procedures. However conventional PNL is done through the tract size $>24$ Fr. Thus, mini-PNL terminology is used for the PNL procedures done through 22-14 Fr-tract size (Turk et al. 2015). On the other hand, length of mini PNL instruments may not be long as to reach to desired calyx or to allow manipulations in the kidney, in obese patients. To the best of our knowledge, no previous study specifically investigated the safety or efficacy of mini-PNL in obese subjects. In this study, we aimed to compare the safety and efficacy of mini-PNL procedure between nonobese and obese patients.

\section{Methods}

One hundred and eighty-two mini-PNL procedures were performed between May 2013 and January 2015 and their results were evaluated retrospectively. The patients were divided into two groups according to their BMI values. Patients with a BMI value lower than $30 \mathrm{~kg} / \mathrm{m}^{2}$ were defined as the non-obese group, and those with a BMI of $\geq 30 \mathrm{~kg} / \mathrm{m}^{2}$ were defined as the obese group. Patients were evaluated pre-operatively with urinalysis, urine culture, blood counts, intravenous pyelography (IVP) and computed tomography (CT). Appropriate antibiotics were prescribed to patients with positive urine cultures until a sterile urine culture was obtained. Patients younger than 18 years of age and patients with coagulopathy were excluded from our study. The groups were compared according to their pre-operative demographic values and intraoperative and post-operative results. Patients with radio-opaque stones and non-radio-opaque stones were evaluated with kidney-ureter-bladder (KUB) $\mathrm{X}$-ray and/or ultrasonography on post-operative day 2 , respectively and all patients were evaluated regarding success rate with kidney-ureter-bladder (KUB) X-ray and ultrasonography or $\mathrm{CT}$ on the first post-operative month. Patients with complete stone clearance and patients with residual fragments under $4 \mathrm{~mm}$ were accepted as stone free.

\section{Mini-PNL technique}

Under general anesthesia in the lithotomy position, a 5-Fr ureteral catheter was inserted and fixed to the 16-Fr bladder catheter. The calyceal system was visualized using contrast media in the prone position. Access was performed using an 18-G percutaneous access needle under the fluoroscopy. A 0.035 -inch hydrophilic guidewire was inserted, and a second guidewire was inserted with a dual lumen catheter. Dilatation was performed using Amplatz dilatators up to $18 \mathrm{Fr}$. After placement Amplatz sheath to the collecting system over the dilator, nephroscopy was done with 12 or 17 Fr nephroscope (Karl Storz, Tuttlingen, Germany) and stone fragmentation was performed using a laser, pneumatic or ultrasonic lithotripter. Stone extraction was performed using a 5-Fr stone extraction device. The surgery was finalized after insertion of a 14-Fr nephrostomy tube under fluoroscopy.

\section{Statistical analysis}

The variables were expressed as numbers, percentage, mean values, and standard deviations. Comparisons were performed by Chi square test, Student $t$ test, and Mann-Whitney U test. The significance of the relationships between the variables was analyzed using Pearson correlation analysis.

\section{Results}

Pre-operative demographic values are shown in Table 1. The non-obese group consisted of 133 and the obese group consisted of 49 patients. The subjects in the nonobese group were younger $(\mathrm{p}<0.001)$. Average stone size was similar between the groups $(23.9 \pm 9 \mathrm{~mm}$ and $26.2 \pm 8.6$ in the non-obese and obese groups, respectively, $\mathrm{p}=0.13$ ). There was no significant difference between two groups in creatinine values, stone locations, operation side, hydronephrosis grade, opacity of stones, and history of operations and SWL.

Intra-operative findings of groups were summarized in Table 2. There was no statistical difference between two groups in operation time, fluoroscopy time, number of accesses, and access sites. Mean operation time was $111 \mathrm{~min}$ in the non-obese group and $109 \mathrm{~min}$ in the obese group $(\mathrm{p}=0.805)$. Stone-free rates were $70.7 \%$ in the non-obese group and $71.4 \%$ in the obese group $(\mathrm{p}=0.9)$. There was no significant difference in length of hospital stay, hemoglobin drop, or post-operative complications (Table 3). Body mass index was not correlated with any of the study variables (Table 4).

\section{Discussion}

Obesity appears to be a serious health problem in multiple ways and its incidence is significantly rising in the last 50 years. Risk of renal stone disease is high in obese 
Table 1 Comparison of pre-operative values

\begin{tabular}{|c|c|c|c|c|c|}
\hline & \multicolumn{4}{|l|}{ Body Mass Index } & \multirow[t]{3}{*}{$p$ values } \\
\hline & \multicolumn{2}{|l|}{$<30$} & \multicolumn{2}{|l|}{$\geq 30$} & \\
\hline & & $\%$ & & $\%$ & \\
\hline Number of patients & 133 & & 49 & & \\
\hline Sex & & & & & 0.004 \\
\hline Male & 91 & 68.4 & 22 & 44.9 & \\
\hline Female & 42 & 31.6 & 27 & 55.1 & \\
\hline Mean age (years) & $41.8 \pm 12.6(18-78)$ & & $51.7 \pm 12.5(19-76)$ & & $<0.001$ \\
\hline Mean Body Mass Index $\left(\mathrm{kg} / \mathrm{m}^{2}\right)$ & $25.5 \pm 4.3(17.8-29.8)$ & & $34.3 \pm 4.3(30.0-46.4)$ & & $<0.001$ \\
\hline Mean stone size $(\mathrm{mm})$ & $23.9 \pm 9.0(5-60)$ & & $26.2 \pm 8.6(15-60)$ & & 0.134 \\
\hline \multicolumn{6}{|l|}{ Localization of stones } \\
\hline Multiple calyces & 67 & 50.4 & 27 & 55.1 & \\
\hline Pelvis & 25 & 18.8 & 8 & 16.3 & \\
\hline Lower calyces & 29 & 21.8 & 12 & 24.5 & \\
\hline Middle calyces & 7 & 5.3 & 0 & 0 & \\
\hline Upper calyces & 5 & 3.8 & 2 & 4.1 & \\
\hline \multicolumn{6}{|l|}{ Side of the operated kidney } \\
\hline Left & 72 & 54.1 & 30 & 61.2 & \\
\hline Right & 61 & 45.9 & 19 & 38.8 & \\
\hline Grade of hydronephrosis & & & & & 0.802 \\
\hline 0 & 4 & 3 & 1 & 2.1 & \\
\hline 1 & 76 & 57.6 & 31 & 64.6 & \\
\hline 2 & 44 & 33.3 & 15 & 31.3 & \\
\hline 3 & 7 & 5.3 & 1 & 2.1 & \\
\hline 4 & 1 & 0.8 & 0 & 0 & \\
\hline Opacity & & & & & 0.050 \\
\hline Non-opaque & 3 & 2.3 & 4 & 8.2 & \\
\hline Opaque & 129 & 97 & 43 & 87.8 & \\
\hline Semi-opaque & 1 & 0.8 & 2 & 4.1 & \\
\hline Previous ESWL history & 29 & 23.6 & 17 & 37.0 & 0.082 \\
\hline Previous PNL history & 46 & 37.1 & 20 & 46.5 & 0.277 \\
\hline Previous open surgery history & 26 & 21 & 8 & 17.8 & 0.648 \\
\hline
\end{tabular}

BMI Body Mass Index

patients due to hyperinsulinemia related acidic urine and disordered ammonium metabolism and disorders of renal tubules (Asplin 2009; Sakhaee and Maalouf 2008).

Shock wave lithotripsy, F-URS, open surgery, PNL and mini-PNL are treatment options for renal stone disease. SWL is not a reasonable option in obese patients due to increased skin-to-stone distance, inability to maintain a clear vision during fluoroscopy, and technical difficulties in positioning of the patient (Robert et al. 1999; Munoz et al. 2003; Alkan et al. 2015). Open surgery is not routinely preferred due to increased risk of surgery site infections, post-operative pain, thromboembolism, and respiratory problems; prolonged healing time; and longer incision and scar formation (Choban and Flancbaum 1997).
Recently, F-URS is an outstanding treatment method due to its natural orifice transluminal endoscopic surgery (NOTES) feature. F-URS has been shown to be effective in treatment of kidney stones, which are less than $2 \mathrm{~cm}$ but operation time is longer and multiple operations may be needed needed for complete stone clearance (Nguyen and Belis 1998; Sergeyev et al. 2007). These problems limit the usage of F-URS in obese patients because prolonged operation times and multiple procedures can increase the risk of morbidity and mortality in these subjects (Oberg and Poulsen 1996; Adams and Murphy 2000; Bond 1993; Postlethwait and Johnson 1972; Perberton and Manax 1971).

Notably, PNL is a preferable method in the treatment of renal stone disease in obese patients compared with 
Table 2 Comparison of intra-operative values

\begin{tabular}{|c|c|c|c|c|c|}
\hline & \multicolumn{4}{|l|}{ BMI } & \multirow[t]{3}{*}{ p values } \\
\hline & \multicolumn{2}{|l|}{$<30$} & \multicolumn{2}{|l|}{$\geq 30$} & \\
\hline & & $\%$ & & $\%$ & \\
\hline Number of patients & 133 & & 49 & & \\
\hline Mean operation time (minutes) & $111.4 \pm 42.5(45-250)$ & & $109.6 \pm 41.6(40-240)$ & & 0.805 \\
\hline Mean fluoroscopy time (minutes) & $5.4 \pm 3.4(0.7-18.8)$ & & $5.8 \pm 4.7(0.8-20)$ & & 0.471 \\
\hline Number of access & & & & & 0.415 \\
\hline 1 & 123 & 92.5 & 43 & 87.8 & \\
\hline 2 & 9 & 6.8 & 6 & 12.2 & \\
\hline 3 & 1 & 0.8 & 0 & 0 & \\
\hline Access site & & & & & 0.486 \\
\hline Multiple & 7 & 5.4 & 4 & 8.2 & \\
\hline Lower calyces & 105 & 78.9 & 41 & 83.7 & \\
\hline Middle calyces & 17 & 12.8 & 3 & 6.1 & \\
\hline Upper calyces & 4 & 0.8 & 1 & 2.0 & \\
\hline
\end{tabular}

BMI Body Mass Index

Table 3 Comparison of post-operative values

\begin{tabular}{|c|c|c|c|c|c|}
\hline & \multicolumn{4}{|l|}{ BMI } & \multirow[t]{3}{*}{$p$ values } \\
\hline & \multicolumn{2}{|l|}{$<30$} & \multicolumn{2}{|l|}{$\geq 30$} & \\
\hline & & $\%$ & & $\%$ & \\
\hline Number of patients & 133 & & 49 & & \\
\hline Mean hospitalization duration (hours) & $65.7 \pm 28.8(24-192)$ & & $61.3 \pm 47.1(24-192)$ & & 0.454 \\
\hline Mean hemoglobin drop (g/dl) & $0.78 \pm 1.12(0-4.8)$ & & $1.08 \pm 1.2(0-4.2)$ & & 0.139 \\
\hline \multicolumn{6}{|l|}{ Post-operative complications } \\
\hline Fever & 1 & 0.8 & 1 & 2.0 & 0.459 \\
\hline Transfusion & 4 & 3.0 & 1 & 2.0 & 0.723 \\
\hline Angioembolisation & 1 & 0.8 & 0 & 0 & 0.543 \\
\hline Pulmonary complications & 0 & & 0 & & \\
\hline Post-operative DJ insertion & 24 & 18 & 6 & 12.2 & 0.350 \\
\hline Results & & & & & 0.921 \\
\hline Number of patients with residual stones & 39 & 29.3 & 14 & 28.6 & \\
\hline Number patients with stone-free status & 94 & 70.7 & 35 & 71.4 & \\
\hline
\end{tabular}

$B M I$ Body Mass Index

other treatment modalities. But it is clear that there are certain difficulties of the PNL procedure, especially in obese patients. Difficulty of positioning of the patient in the prone position, increased risk of pressure ulcer formation, and the potential need of increased rates of transfusion can be listed as some of these difficulties.

Mini-PNL, which was first defined for pediatric population by Helal et al. (1997), is a new treatment option for obese patients, with its lower risk of parenchymal injury and smaller tract size. There are no prior studies in literature about safety and efficacy of mini-PNL in obese patients. In a CROES study published by Fuller et al. (2012), conventional PNL was performed in 5803 patients who were stratified into 4 different groups according to their BMIs. Stone-free rates were $78.9 \%$ in the obese group and $65.6 \%$ in the morbid obese group. In a different study about efficacy of F-URS in obese patients published by Sari et al. (2013), stone-free rates were $73.6 \%$ in obese and $61.5 \%$ in morbid obese group. In a similar study about efficacy of F-URS in obese patients with stones greater than $2 \mathrm{~cm}$ published by Alkan et al. (2015), stone-free rates were $75.6 \%$ in obese and $66.6 \%$ in morbid obese patients. In a similar study by Carson et al. (1988), 44 obese and 226 non-obese patients 
Table 4 Summary of univariate and multivariate anaylsis, which shows the effect of obesity on operation outcomes

\begin{tabular}{lll}
\hline & BMI & p values \\
\hline Mean operation time (min) & 0.805 & 0.486 \\
Mean fluoroscopy time (min) & 0.471 & 0.635 \\
Mean hospitalization duration (h) & 0.454 & 0.782 \\
Mean hemoglobin drop (g/dl) & 0.139 & 0.043 \\
Post-operative complications & & \\
Fever & 0.462 & 0.638 \\
Transfusion & 0.725 & 0.955 \\
Angioembolisation & 0.545 & 0.473 \\
Post-operative DJ insertion & 0.352 & 0.917 \\
Result & 0.922 & 0.54 \\
\hline
\end{tabular}

BMI Body Mass Index

were compared and no statistical difference was identified in operation times, the need for multiple procedures to achieve complete clearance, total length of hospital stay, complication rates, or stone-free rates. In our study, stone-free rates were $70.7 \%$ in the non-obese group and $71.4 \%$ in the obese group.

In a CROES study about conventional PNL by Fuller et al. (2012), complication rates in obese and morbid obese patients were 18.5 and $22.1 \%$, respectively. In a study by Simsek et al. (2014), conventional PNL was performed in 2012 patients and these patients were stratified into 4 groups according to their BMIs. 370 patients had a BMI value greater than $30 \mathrm{~kg} / \mathrm{m}^{2} .20$ patients $(5.4 \%)$ developed pelvicalyceal perforation, 5 patients had pulmonary problems (1.3\%), 2 patients developed cardiac problems $(0.5 \%)$, and 1 patient experienced hemorrhage, which required nephrectomy $(0.2 \%)$. In our study, the complication rate was $12.2 \%$ in the obese group and pelvicalyceal perforation was seen in 4 patients $(2.1 \%)$. There were no pulmonary complications or hemorrhage requiring nephrectomy.

Bleeding is a frequent complication of PNL and management of bleeding is crucial. According to studies that have included large patient series, bleeding which requires transfusion can be seen in 5-18\% of cases and bleeding which requires embolization can be seen in 0.3-1 \% (Michel et al. 2007; Skolarikos and de la Rosette 2008; Kessaris et al. 1995; Turna et al. 2007; ElNahas et al. 2007). Imaging modality during percutaneous access (ultrasonography vs. fluoroscopy), tract dilatation technique, tract size, and tract length are all factors, which can affect bleeding rates in PNL (Pearle et al. 1998). In the light of this information, mini-PNL seems to be a preferable option due to its lesser parenchymal injury formation. In a study by Ates et al. (2011), evaluated the safety of PNL on 194 patients and bleeding rates in obese and non-obese patients were 12.3 and $12.9 \%$, respectively. In the CROES study, conventional PNL was performed in patients and bleeding rates in obese and morbid obese patients were 4.5 and $5.2 \%$, respectively (Fuller et al. 2012). In our study, mean hemoglobin drop in the non-obese and obese groups were 0.78 and $1.08 \mathrm{mg} / \mathrm{dl}$, respectively. The rates of transfusion requirements were similarly low in the two groups. Transfusion rates and bleeding rates in our study were lower when compared to conventional PNL data in the literature.

Our study has several limitations, including the retrospective design of the study, presence of multiple surgeons instead of a single surgeon, absence of stone analysis results and evaluation of stone-free status. It is well known that KUB and ultrasonography are not as sensitive as computed tomography in the detection of residual stone fragments, especially in obese subjects. Although CT examination would provide a more accurate success rate in obese subjects, the stone-free status was assessed with KUB and ultrasonography due to concerns about the radiation exposure.

\section{Conclusions}

Our study has demonstrated that efficacy and safety of the mini-PNL procedure were similar in obese and nonobese groups. Mini-PNL procedure seems to be a safe and efficacious option in obese patients with appropriate sized stones.

\section{Authors' contributions \\ FA conceived the study and wrote the manuscript. OK, EK and FO participated in interpretation of the data. BU, FY participated in editing the language of manuscript. BA and OK performed the statistical analysis and participated in interpretation of the data. AS, MB and GG participated in the coordination of the study and helped to review the paper. FA, FO, AE prepared reply of revi- sion. All authors read and approved the final manuscript.}

\section{Acknowledgements}

The authors appreciate reviewers and editor for their valuable comments and suggestions towards improving the quality of the manuscript.

\section{Competing interests}

The authors declare that have no competing interests.

\section{Ethical approval}

All procedures performed in studies involving human participants were in accordance with the ethical standards of the institutional and/or national research committee and with the 1964 Helsinki declaration and its later amendments or comparable ethical standards. (Haseki Training and Research Hospital Ethical Committee No: 304, 2015).

\section{Informed consent}

Informed consent was obtained from all individual participants included in the study.

Received: 12 February 2016 Accepted: 14 July 2016

Published online: 22 July 2016 


\section{References}

Adams JP, Murphy PG (2000) Obesity in anaesthesia and intensive care. Br J Anaesth 85:91-108

Al-Hayek S, Schwen ZR, Jackman SV, Averch TD (2013) The impact of obesity on urine composition and nephrolithiasis management. J Endourol 27:379-383

Alkan E, Arpali E, Ozkanli AO, Basar MM, Acar O, Balbay MD (2015) RIRS is equally efficient in patients with different BMI scores. Urolithiasis $43: 243-248$

Asplin JR (2009) Obesity and urolithiasis. Adv Chronic Kidney Dis 16:11-20

Ateş M, Karalar M, Tüzel E, Pektaş F, Yıldırım B (2011) Safety and efficacy of percutaneous nephrolithotomy in obese patients. Turk J Urol 37:105-111

Bagrodia A, Gupta A, Raman JD, Bensalah K, Pearle MS, Lotan Y (2008) Impact of body mass index on cost and clinical outcomes after percutaneous nephrostolithotomy. Urology 72:756-760

Bond A (1993) Obesity and difficult intubation. Anaesth Intensiv Care 21:828-830

Carson CC, Danneberger JE, Weinerth JL (1988) Percutaneous lithotripsy in morbid obesity. J Urol 139:243-245

Choban PS, Flancbaum L (1997) The impact of obesity on surgical outcomes: a review. J Am Coll Surg 185:593-603

Curhan GC (2007) Epidemiology of stone disease. Urol Clin North Am 34:287-293

Curhan GC, Willett WC, Rimm EB, Speizer FE, Stampfer MJ (1998) Body size and risk of kidney stones. J Am Soc Nephrol 9:1645-1652

Desai MR, Kukreja RA, Desai MM et al (2004) A prospective randomized comparison of type of nephrostomy drainage following percutaneous nephrostolithotomy: large bore versus small bore versus tubeless. J Uro 172:565-567

El-Assmy AM, Shokeir AA, El-Nahas AR et al (2007) Outcome of percutaneous nephrolithotomy: effect of body mass index. Eur Urol 52:199-204

El-Nahas AR, Shokeir AA, El-Assmy AM et al (2007) Postpercutaneous nephrolithotomy extensive hemorrhage: a study of risk factors. J Urol 177:576-579

Fabrizio MD, Behari A, Bagley DH (1998) Ureteroscopic management of intrarenal calculi. J Urol 159:1139-1143

Faerber GJ, Goh M (1997) Percutaneous nephrolithotripsy in the morbidly obese patient. Tech Urol 3:89-95

Fuller A, Razvi H, John DD et al (2012) The CROES Percutaneous Nephrolithotomy Global Study: the Influence of Body Mass Index on Outcome on behalf of the CROES PCNL Study Group. J Urol 188:138-144

Goldfarb DS (2003) Increasing prevalence of kidney stones in the United States. Kidney Int 63:1951-1952

Helal M، BlackT, Lockhart J, Figueroa TE (1997) The Hickman peel-away sheath: alternative for pediatric percutaneous nephrolithotomy. J Endourol 11:171-172

Kessaris DN, Bellman GC, Pardalidis NP, Smith AG (1995) Management of hemorrhage after percutaneous renal surgery. J Urol 153:604-608

Koo BC, Burtt G, Burgess NA (2004) Percutaneous stone surgery in the obese: outcome stratified according to body mass index. BJU Int 93:1296-1299

Kukreja R, Desai M, Patel S, Bapat S, Desai M (2004) Factors affecting blood loss during percutaneous nephrolithotomy: prospective study. J Endourol 18:715-722
Michel MS, Trojan L, Rassweiler JJ (2007) Complications in percutaneous nephrolithotomy. Eur Urol 51:899-906

Munoz RD, Tirolien PP, Belhamou S et al (2003) Treatment of reno-ureteral lithiasis with ESWL in obese patients. Apropos of 150 patients. Arch Esp Urol 56:933-938

Nguyen TA, Belis JA (1998) Endoscopic management of urolithiasis in the morbidly obese patient. J Endourol 12:33-35

Oberg B, Poulsen TD (1996) Obesity: an anaesthetic challenge. Acta Anaesthesiol Scand 40:191-200

Olbert PJ, Hegele A, Schrader AJ, Scherag A, Hofmann R (2007) Pre- and perioperative predictors of short-term clinical outcomes in patients undergoing percutaneous nephrolitholapaxy. Urol Res 35:225-230

Pearle MS, Nakada SY, Womack JS, Kryger JV (1998) Outcomes of contemporary percutaneous nephrostolithotomy in morbidly obese patients. J Urol 160:669-673

Perberton LB, Manax WG (1971) Relationship of obesity to postoperative complications after cholecystectomy. Am J Surg 121:87-90

Postlethwait RW, Johnson WD (1972) Complications following surgery for duodenal ulcer in obese patients. Arch Surg 105:438-440

Robert M, A'Ch S, Lanfrey P, Guiter J, Navratil H (1999) Piezoelectric shockwave lithotripsy of urinary calculi: comparative study of stone depth in kidney and ureter treatments. J Endourol 13:699-703

Sakhaee K, Maalouf NM (2008) Metabolic syndrome and uric acid nephrolithiasis. Semin Nephrol 28:174-180

Sari E, Tepeler A, Yuruk E et al (2013) Effect of the body mass index on outcomes of flexible ureterorenoscopy. Urolithiasis 41:499-504

Sergeyev I, Koi PT, Jacobs SL, Godelman A, Hoenig DM (2007a) Outcome of percutaneous surgery stratified according to body mass index and kidney stone size. Surg Laparosc Endosc Percutan Tech 17:179-183

Sergeyev I, Koi PT, Jacobs SL, Godelman A, Hoenig DM (2007b) Outcome of percutaneous surgery stratified according to body mass index and kidney stone size. Surg Laparosc Endosc Percutan Tech. 17:179-183

Shikora SA (2005) Severe obesity: a growing health concern A.S.P.E.N. should not ignore. JPEN 29:288-297

Simşek A, Özgör F, Akbulut MF et al (2014) Does bodymass index effect the success of percutaneous nephrolithotomy? Turk J Urol 40:104-109

Skolarikos A, de la Rosette J (2008) Prevention and treatment of complications following percutaneous nephrolithotomy. Curr Opin Urol 18:229-234

Stamatelou KK, Francis ME, Jones CA, Nyberg LM, Curhan GC (2003) Time trends in reported prevalence of kidney stones in the United States, 1976-1994. Kidney Int 63:1817-1823

Taylor EN, Stampfer MJ, Curhan GC (2005) Obesity, weight gain, and the risk of kidney stones. JAMA 293:455-462

Thomas R, Cass AS (1993) Extracorporeal shockwave lithotripsy in morbidly obese patients. J Urol 150:30-32

Turk C, Knoll T, Petrik A et al (2015) EAU guidelines on urolithiasis. Eur Urol 1-71

Turna B, Nazli O, Demiryoguran S, Mammadov R, Cal C (2007) Percutaneous nephrolithotomy: variables that influence hemorrhage. Urology 69:603-607

Yang RM, Bellman GC (2004) Tubeless percutaneous renal surgery in obese patients. Urology 63:1036-1041

\section{Submit your manuscript to a SpringerOpen ${ }^{\odot}$ journal and benefit from:}

- Convenient online submission

- Rigorous peer review

- Immediate publication on acceptance

- Open access: articles freely available online

- High visibility within the field

- Retaining the copyright to your article

Submit your next manuscript at springeropen.com 